\title{
CALCÁRIO E POTÁSSIO PARA A CULTURA DE SOJA
}

\author{
Hipólito Assunção Antonio Mascarenhas ${ }^{1,5 *}$; Roberto Tetsuo Tanaka ${ }^{1,5}$; Quirino Augusto de \\ Camargo Carmello²; Paulo Boller Gallo ${ }^{3}$; Gláucia Maria Bovi Ambrosano ${ }^{4}$ \\ ${ }^{1}$ Centro de Plantas Graníferas - IAC, C.P. 28 - CEP: 13001-970 - Campinas, SP. \\ ${ }_{3}^{2}$ Depto. de Solos e Nutrição de Plantas - USP/ESALQ, C.P. 9 - CEP: 13418-900 - Piracicaba, SP. \\ ${ }^{3}$ Núcleo Experimental - IAC, C.P. 58 - CEP: 13730-970 - Mococa, SP. \\ ${ }_{5}^{4}$ Faculdade de Odontologia - UNICAMP, C.P. 52 - CEP:13414-018 - Piracicaba, SP. \\ ${ }^{5}$ Bolsista CNPq. \\ *Autor correspondente<hipolito@cec.iac.br>
}

RESUMO: Durante três anos agrícolas foi conduzido um experimento em Latossolo Vermelho-Escuro álico, textura argilosa em Mococa, SP, para estudar o efeito da aplicação de doses de calcário dolomítico (0, 3,5 e 7 $\left.\mathrm{t} \mathrm{ha}^{-1}\right)$ e de potássio $\left(0,150,300,450\right.$ e $600 \mathrm{~kg} \mathrm{ha}^{-1}$ de $\mathrm{K}_{2} \mathrm{O}$ na forma de $\left.\mathrm{KCl}\right)$ sobre a produtividade da soja. Os dois insumos foram aplicados a lanço e incorporados ao solo. Foram utilizados os cultivares IAC-17 e FT-2, ambos com 110 dias de ciclo, cujas sementes foram inoculadas com Bradyrhizobium fixador do $\mathrm{N}$ atmosférico. $\mathrm{Na}$ análise conjunta, verificou-se que a máxima eficiência técnica seria obtida com 383 e $441 \mathrm{~kg} \mathrm{ha}^{-1}$ de $\mathrm{K}_{2} \mathrm{O}$, em associação, respectivamente, com 3,5 e $7 \mathrm{t} \mathrm{ha}^{-1}$ de calcário, e também quando as relações de $(\mathrm{Ca}+\mathrm{Mg}) /$ $\mathrm{K}$ foram, em média, de 14 a 23 no solo. A produtividade do cv. FT-2 sempre foi maior do que a do IAC-17, exceto na ausência da adubação potássica. As produtividades máximas de 2384 e $1786 \mathrm{~kg} \mathrm{ha}^{-1}$, foram obtidas para os cultivares FT-2 e IAC-17 com a adubação de 450 e $370 \mathrm{~kg} \mathrm{ha}^{-1}$ de $\mathrm{K}_{2} \mathrm{O}$, respectivamente. Com os mesmos $370 \mathrm{~kg} \mathrm{ha}^{-1}$ de $\mathrm{K}_{2} \mathrm{O}$ aplicados no cv. IAC-17, seriam obtidos $2354 \mathrm{~kg} \mathrm{ha}^{-1} \mathrm{com} \mathrm{o} \mathrm{cv.} \mathrm{FT-2,} \mathrm{o} \mathrm{que} \mathrm{é}$ indicativo da maior eficiência deste genótipo na utilização do nutriente potássico aplicado.

Palavras-chave: Glycine max, produção, cultivar, relação de cátions

\section{LIME AND POTASSIUM FOR THE SOYBEAN CROP}

\begin{abstract}
An experiment was carried out on analic Dark Red Latosol in Mococa, SP, Brazil, during three years, to study the effects of the application of dolomitic limestone $\left(0,3.5\right.$ and $\left.7 \mathrm{tha}^{-1}\right)$ and of potassium $(0,150$, 300,450 and $600 \mathrm{~kg} \mathrm{ha}^{-1}$ of $\mathrm{K}_{2} \mathrm{O}$ in the form of $\mathrm{KCl}$ ) broadcasted. Two soybean cultivars, IAC-17 and FT-2, of 110 day cycle were utilized, the latter being more demanding for potassium. The results of three crops showed that in the absence of liming there is no response to potassium. Maximum yields were obtained at the rate of 383 and $441 \mathrm{~kg} \mathrm{ha}^{-1}$ of $\mathrm{K}_{2} \mathrm{O}$, respectively in the presence of 3.5 and $7.0 \mathrm{t} \mathrm{ha}^{-1}$ of dolomitic limestone, where the soil ratio $(\mathrm{Ca}+\mathrm{Mg}) / \mathrm{K}$ were in average between 14 and 23 . The $\mathrm{cv}$. FT-2 had always a higher yield than IAC-17, except when no potassium was applied. Cultivars FT-2 and IAC-17 produced maximum grain yields of 2384 and $1786 \mathrm{~kg} \mathrm{ha}^{-1}$ with the application of 450 and $370 \mathrm{~kg} \mathrm{ha}^{-1}$ of $\mathrm{K}_{2} \mathrm{O}$, respectively. At the $370 \mathrm{~kg} \mathrm{ha}^{-1}$, FT-2 produced a yield of $2354 \mathrm{~kg} \mathrm{ha}^{-1}$ of soybean grains, showing that it is more efficient in utilizing the potassium fertilizer in comparison to cultivar IAC-17.
\end{abstract}

Key words: Glycine max, yield, cultivars, cation relationship

\section{INTRODUÇÃO}

Após o nitrogênio, o potássio é o nutriente absorvido em maior quantidade pelas plantas, exportando até $18,5 \mathrm{~kg} \mathrm{t}^{-1}$ de grãos de soja (Tanaka \& Mascarenhas, 1992). O potássio nativo é uma das principais fontes para as plantas. Gargantini et al. (1970) em levantamento de fertilidade dos solos do Estado de São Paulo, observaram a ocorrência de teores variados entre médio e alto. Entretanto, nas décadas de 60 e 70, os experimentos com soja conduzidos com níveis de potássio em diferentes solos do Estado de São Paulo mostraram respostas inconsistentes (Mascarenhas et al., 1971).

No início da década de 80 , foram observados os primeiros sintomas de deficiência de potássio em culturas comerciais de soja. Os sintomas eram clorose nas folhas, hastes verdes, retenção foliar e formação de frutos partenocárpicos (Mascarenhas et al., 1987;
Raij \& Mascarenhas, 1976). Os estudos mostraram que esses sintomas foram devidos ao uso de cultivares com maior exportação de nutrientes por área e portanto com maior exigência nutricional e também à aplicação de adubos com teores baixos de potássio.

Os experimentos de longa duração para avaliar o efeito direto e/ou residual de doses de $\mathrm{K}$ em soja, realizados nos estados de São Paulo, Paraná e Santa Catarina, mostraram que as concentrações daquele nutriente diminuíram tanto no solo como nas folhas, com os cultivos, afetando negativamente a produtividade (Mascarenhas et al., 1981; Rosolem et al., 1984; Yamada \& Borkert 1992; Borkert et al., 1997; Scherer, 1998).

Por outro lado, quando a disponibilidade de $\mathrm{Ca} e$ de $\mathrm{Mg}$ aumentam em relação à de $\mathrm{K}$, devido à calagem, a absorção deste último pelas plantas é reduzida pela competição entre os três cátions. Entretanto, a calagem pode aumentar a disponibilidade de $\mathrm{K}$ no solo 
aumentando assim a sua absorção pela planta (Jaworski \& Barber, 1957).

Essa relação entre os nutrientes foi analisada por Mascarenhas et al. (1987), em mais de 100 amostras de solo de campos de produção de soja. Os autores observaram que a alta produtividade de grãos foi obtida quando o índice de $(\mathrm{Ca}+\mathrm{Mg}) / \mathrm{K}$ no solo estava entre 23 e 31, comprovado também por Rosolem et al. (1992).

O objetivo deste estudo foi o de avaliar o efeito da aplicação de doses de calcário e de potássio sobre a produtividade de cultivares de soja com eficiência diferenciada na utilização do potássio no solo.

\section{MATERIAL E MÉTODOS}

O experimento foi conduzido no Núcleo Experimental de Mococa do IAC num Latossolo Vermelho Escuro álico A moderado textura argilosa, que apresentava as seguintes características químicas: $\mathrm{pH}\left(\mathrm{CaCl}_{2}\right)=4,2$; M.O. $=22 \mathrm{~g} \mathrm{dm}^{-3} ; \mathrm{P}$ extraído com resina $=44 \mathrm{mg} \mathrm{dm}^{-3} ; \mathrm{K}=0,7$ $\mathrm{mmol}_{\mathrm{c}} \mathrm{dm}^{-3} ; \mathrm{Ca}=7 \mathrm{mmol}_{\mathrm{c}} \mathrm{dm}^{-3} ; \mathrm{Mg}=3 \mathrm{mmol}_{\mathrm{c}} \mathrm{dm}^{-3}, \mathrm{CTC}=$ $71,7 \mathrm{mmol}_{\mathrm{c}} \mathrm{dm}^{-3} ; \mathrm{H}+\mathrm{Al}=61 \mathrm{mmol}_{\mathrm{c}} \mathrm{dm}^{-3}$ e V\%= 15 .

Os tratamentos foram distribuídos em delineamento experimental de blocos ao acaso, em esquema de parcelas subsubdivididas, com quatro repetições. Nas parcelas $(15 \mathrm{~m} \times 5 \mathrm{~m})$ foram testadas as doses de calcário dolomítico $\left(0,3,5\right.$ e $\left.7 \mathrm{t} \mathrm{ha}^{-1}\right)$, nas subparcelas ( $5 \mathrm{~m} \times 3 \mathrm{~m})$, as doses de potássio $(0,150$, 300,450 e $600 \mathrm{~kg} \mathrm{ha}^{-1}$ de $\mathrm{K}_{2} \mathrm{O}$ ), e nas subsubparcelas ( 5 $\mathrm{m} \times 1,5 \mathrm{~m}$ ), dois cultivares de soja precoce (IAC-17 e FT2 ), ambos de 110 dias de ciclo. As sementes foram inoculadas com a bactéria Bradyrhizobium japonicum. No primeiro ano, foi estudado o efeito direto e, nos dois subsequentes, o efeito residual.

Em junho de 1991, o calcário foi aplicado a lanço, e incorporado na camada de $0-20 \mathrm{~cm}$ do solo. O cloreto de potássio foi também aplicado a lanço um mês antes da semeadura e incorporado com enxada rotativa. $O$ fósforo foi aplicado anualmente no sulco, na dose de 60 $\mathrm{kg} \mathrm{ha}^{-1}$ de $\mathrm{P}_{2} \mathrm{O}_{5}$, na forma de superfosfato simples.

As doses de calcário foram determinadas em função dos resultados obtidos por Gallo et al. (1986), de uma área adjacente deste ensaio, em que $4 \mathrm{t} \mathrm{ha}^{-1}$ foi considerada a mais adequada para a máxima eficiência técnica. As doses de potássio foram estabelecidas em função da ausência de resposta da soja ao mesmo, em aplicações no sulco da semeadura (Mascarenhas et al. 1976 e Raij \& Mascarenhas, 1976). A escolha dos cultivares de soja foi efetuada em função da maior exigência em K pelo FT-2 e menor pelo IAC-17.

O acompanhamento do estado nutricional das plantas foi feito com amostragens de folhas realizadas no início do florescimento, colhendo-se 30 folhas maduras por subsubparcela. As amostras simples do solo $(0-20 \mathrm{~cm}) \mathrm{em}$ número de seis foram retiradas das subparcelas logo após a colheita da soja, para representar a composta. Após a secagem e o preparo, as amostras de folhas e de solo foram analisadas utilizando as metodologias propostas por Bataglia et al. (1983) e Raij \& Quaggio (1983), respectivamente.

Os dados obtidos foram submetidos à análise de variância e quando pertinentes, aos desdobramentos dos graus de liberdade das interações significativas aplicandose a regressão. Os ajustes entre as variáveis foram efetuados nas formas linear ou quadrática em função da significância do maior grau.

\section{RESULTADOS E DISCUSSÃO}

Nas duas primeiras safras (1991/92 e 1992/93) a semeadura foi efetuada em novembro, época recomendada para cultivares precoces. A precipitação pluvial foi bem distribuída no primeiro ano, enquanto no segundo houve alta intensidade de chuvas, três semanas antes da maturação. Em 1993/94, a semeadura foi adiada para dezembro devido à seca.

No primeiro ano, a resposta ao potássio foi bem notória em plantas com 45 dias. Nas parcelas que não receberam calagem e adubação potássica, os sintomas de deficiência de $\mathrm{K}$ iniciaram-se no florescimento, com um terço de margens das folhas inferiores apresentando clorose. Setenta e cinco dias após a emergência, as folhas deficientes em potássio ficaram necróticas, semelhantes às descritas por Nelson \& Barber (1964). Os sintomas de deficiência nos dois cultivares também foram observados nos tratamentos com 3,5 e 7 t ha $^{-1}$ de calcário, na ausência de potássio, provavelmente por causa do desequilíbrio entre $(\mathrm{Ca}+\mathrm{Mg}) / \mathrm{K}$, ou outra relação que envolva os três cátions, dois a dois. Sem a calagem, as folhas das plantas estavam amarelecidas, mostrando deficiência de nitrogênio. A altura das plantas e o tamanho das folhas aumentaram progressivamente com o potássio e a calagem quando aplicados em doses equilibradas.

Com relação à produtividade de soja, houve uma interação significativa entre os fatores estudados, independente dos cultivares, durante os três anos. A TABELA 1 apresenta todas as equações ajustadas à forma quadrática e significativa entre doses de potássio em função de cada dose de calcário para a variável produtividade de soja.

Em 1991/92, em ausência de calcário, o efeito do potássio foi linear e não significativo. Em presença de 3,5 e $7 \mathrm{t} \mathrm{ha}^{-1}$ de calcário, houve efeito, mas a dose de máxima produtividade seriam obtidas com 829 e $626 \mathrm{~kg} \mathrm{ha}^{-1}$ de $\mathrm{K}_{2} \mathrm{O}$, extrapolando a dose máxima de potássio utilizada no experimento.

No primeiro ano de efeito residual (1992/93), as plantas dos tratamentos que não receberam adubação potássica mostraram sintomas de ataque do fungo Phomopsis (Ito et al., 1994), atribuídos à menor disponibilidade de K. Essa condição foi acentuada provavelmente devido à lixiviação do elemento causada pelas chuvas intensas no ano anterior, na época da maturação. Apesar daquela doença não ter reduzido a 
produtividade, ela afetou a qualidade fisiológica das sementes, também observado por Ritchey et al. (1987). Nesse ano, em ausência de calagem, o efeito foi linear e, à medida que se aumentou a dose de potássio, houve efeito negativo na produtividade de grãos. Em presença de calagem nas doses de 3,5 e 7,0 t ha ${ }^{-1}$, o efeito da adubação potássica foi quadrático, e as máximas produtividades, de 1775 e $2821 \mathrm{~kg} \mathrm{ha}^{-1}$ seriam alcançadas com doses, respectivamente, de 302 e $335 \mathrm{~kg} \mathrm{ha}^{-1}$ de $\mathrm{K}_{2} \mathrm{O}$ (TABELA 1).

No terceiro ano (1993/94), houve decréscimo na produtividade de grãos de soja. Isso pode ter ocorrido devido à ocorrência de baixas temperaturas $\left(16^{\circ} \mathrm{C}\right)$ durante uma semana, no estádio $R_{2}$ da escala de Fehr et al. (1971), também observada por Tanaka et al. (1993) e elevada precipitação pluvial no período da maturação. Nesse ano, houve uma incidência intensa de Phomopsis (Ito et al., 1994), devido à alta umidade e um ataque leve de Cercospora kikuchii (Ito et al., 1993). Isso ocorreu apenas nos tratamentos onde se utilizaram baixas doses de potássio, tanto na ausência quanto na presença de calcário. Esses fungos apareceram no fim de ciclo e afetaram a qualidade das sementes, conforme já foi relatado por Mascarenhas et al. (1995). Em ausência de calagem, as plantas não cresceram devido à acidez, o que permitiu uma maior quantidade de ervas daninhas entre as linhas. Isso não foi observado nos tratamentos com doses de 300 a $600 \mathrm{~kg} \mathrm{ha}^{-1}$ de $\mathrm{K}_{2} \mathrm{O}$, em presença das doses de calcário, o que permitiu o fechamento de dossel, evitando, assim a entrada de luz e o estabelecimento de plantas infestantes.
Tanto na ausência como na presença de doses de calcário o efeito foi quadrático (TABELA 1, 1993/94). Em ausência de calcário, a máxima eficiência técnica de 1688 $\mathrm{kg} \mathrm{ha}^{-1}$ seria obtida com a dose de $419 \mathrm{~kg} \mathrm{ha}^{-1}$ de $\mathrm{K}_{2} \mathrm{O}$, enquanto com 3,5 e $7 \mathrm{t} \mathrm{ha}^{-1}$, as máximas produtividades de 1866 e $2454 \mathrm{~kg} \mathrm{ha}^{-1}$, seriam obtidas com 385 e 508 $\mathrm{kg} \mathrm{ha}^{-1}$ de $\mathrm{K}_{2} \mathrm{O}$.

Na média de três anos, em ausência de calagem, não houve efeito positivo da aplicação de potássio sobre a produtividade de grãos. Em presença de 3,5 e $7 \mathrm{t} \mathrm{ha}^{-1}$ de calcário, as produtividades máximas de 2094 e 2898 $\mathrm{kg} \mathrm{ha}^{-1}$ seriam obtidas com aplicações, respectivamente, de 383 e $441 \mathrm{~kg} \mathrm{ha}^{-1}$ de $\mathrm{K}_{2} \mathrm{O}$. Verifica-se que houve uma relação direta entre as doses dos fatores estudados para alcançar a máxima eficiência técnica.

Dados de Ritchey et al. (1987) em solo do cerrado mostraram que as maiores produtividades foram obtidas quando a concentração de potássio nas folhas foi superior a $13 \mathrm{gkg}^{-1}$, enquanto, Rosolem et al. (1984) observaram em solos de baixa fertilidade que aquele índice deveria ser superior a $15 \mathrm{~g} \mathrm{~kg}^{-1}$. No presente estudo observou-se que as maiores produtividades de grãos de soja foram obtidas quando as concentrações de potássio nas folhas variaram de 13 a $17 \mathrm{~g} \mathrm{~kg}^{-1}$.

Usherwood (1982) não observou correlações satisfatórias entre os teores $\mathrm{Ca} / \mathrm{K}$ ou $(\mathrm{Ca}+\mathrm{Mg}) / \mathrm{K}$ nos solos e nas folhas para várias culturas. Alvarenga \& Lopes (1988), por outro lado, obtiveram correlações significativas entre $\mathrm{Ca} / \mathrm{Mg}$ na folha e produção de milho, mas não entre $\mathrm{Ca} / \mathrm{K}, \mathrm{Mg} / \mathrm{K}$ ou $(\mathrm{Ca}+\mathrm{Mg}) / \mathrm{K}$. A Figura 1 mostra, independente de cultivar, as tendências das relações

TABELA 1 - Relações quadráticas entre variável independente (dose de $\mathrm{K}_{2} \mathrm{O}$ ) e a variável dependente (produtividade de grãos de soja), fixando-se o fator calcário ou cultivar, e as respectivas doses da produtividade de eficiência física máxima.

\begin{tabular}{|c|c|c|c|c|c|}
\hline Ano & Calcário & Equação & $\mathrm{R}^{2}$ & $\hat{Y}$ máx. & X máx. \\
\hline & ---- t ha-1 ---. & & & \multicolumn{2}{|c|}{-.--- kg ha'-1 -.-.-.. } \\
\hline $1991 / 92$ & 3,5 & $\hat{Y}=1336,5+1,073 X-0,000647 X^{2}$ & 0,891 & 1781 & 829 \\
\hline $1991 / 92$ & 7,0 & $\hat{Y}=1526,2+4,829 X-0,003876 X^{2}$ & 0,981 & 3030 & 626 \\
\hline $1992 / 93$ & 3,5 & $\hat{Y}=1232,4+3,592 X-0,00595 X^{2}$ & 0,960 & 1775 & 302 \\
\hline $1992 / 93$ & 7,0 & $\hat{Y}=1759,1+6,349 X-0,00949 X^{2}$ & 0,875 & 2821 & 335 \\
\hline $1993 / 94$ & 0,0 & $\hat{Y}=1174,8+2,453 X-0,00293 X^{2}$ & 0,967 & 1668 & 419 \\
\hline $1993 / 94$ & 3,5 & $\hat{Y}=1065,9+4,162 X-0,00541 \mathrm{X}^{2}$ & 0,997 & 1866 & 385 \\
\hline $1993 / 94$ & 7,0 & $\hat{Y}=1290,7+4,582 X-0,00451 X^{2}$ & 0,989 & 2454 & 508 \\
\hline Média de 3 anos & 0,0 & $\hat{Y}=1532,4-0,645 X+0,000898 X^{2}$ & 0,952 & 1417 & 359 \\
\hline Média de 3 anos & 3,5 & $\hat{Y}=1325,6+4,017 X-0,00525 X^{2}$ & 0,996 & 2094 & 383 \\
\hline Média de 3 anos & 7,0 & $\hat{Y}=1325,2+5,252-0,00596 \mathrm{X}^{2}$ & 0,998 & 2898 & 441 \\
\hline Ano & Cultivar & Equação & $\mathrm{R}^{2}$ & $\hat{Y}$ máx. & X máx. \\
\hline $1991 / 92$ & FT-2 & $\hat{Y}=1676,3+4,578 X-0,00382 X^{2}$ & 0,999 & 3048 & 599 \\
\hline $1992 / 93$ & FT-2 & $\hat{Y}=1308,1+4,545 \mathrm{X}-0,00651 \mathrm{X}^{2}$ & 0,889 & 2101 & 349 \\
\hline 1993/94 & FT-2 & $\hat{Y}=1290,2+3,699 X-0,00391 X^{2}$ & 0,982 & 2165 & 473 \\
\hline Média de 3 anos & FT-2 & $\hat{Y}=1425,7+4,259 X-0,00473 X^{2}$ & 0,982 & 2384 & 450 \\
\hline $1993 / 94$ & IAC-17 & $\hat{Y}=1065,1+3,761 X-0,00465 X^{2}$ & 0,990 & 1825 & 404 \\
\hline Média de 3 anos & IAC-17 & $\hat{Y}=1296,1+2,645 X-0,00357 X^{2}$ & 0,962 & 1786 & 370 \\
\hline
\end{tabular}

Scientia Agricola, v.57, n.3, p.445-449, jul./set. 2000 


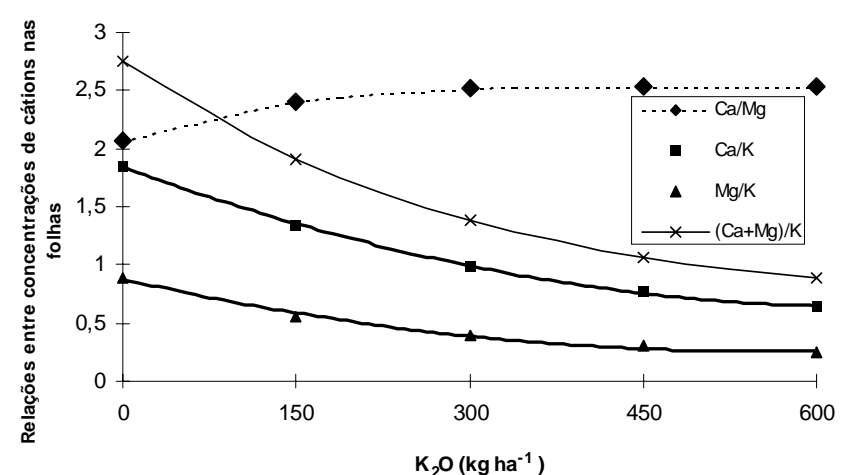

Figura 1 - Relações entre os cátions $\mathrm{Ca}, \mathrm{Mg}$ e $\mathrm{K}$ nas folhas, em presença de doses de $\mathrm{K}_{2} \mathrm{O}$ no solo. Dados médios dos anos e cultivares, em todos níveis de calagem.

$\mathrm{Ca} / \mathrm{Mg}, \mathrm{Ca} / \mathrm{K}, \mathrm{Mg} / \mathrm{K}$ e $(\mathrm{Ca}+\mathrm{Mg}) / \mathrm{K}$ nas folhas em função das doses de adubação potássica. Para a relação $\mathrm{Ca} / \mathrm{Mg}$, pode ser observado um efeito antagônico entre o $\mathrm{K}$ e $\mathrm{o}$ $\mathrm{Mg}$ nas folhas, à medida que o nível de potássio aumentou até $300 \mathrm{~kg} \mathrm{ha}^{-1}$ e nesse nível a relação atingiu 2,52, após a qual não houve mais aumento.

As três relações nas folhas $\mathrm{Ca} / \mathrm{K}, \mathrm{Mg} / \mathrm{K}$ e $(\mathrm{Ca}+$ $\mathrm{Mg}) / \mathrm{K}$, mostraram redução com a aplicação de doses maiores de potássio (Figura 1). Nesta última, houve uma diminuição pronunciada entre as doses de 0 e $300 \mathrm{~kg} \mathrm{ha}^{-1}$ de potássio aplicado, após o que, a diminuição foi menor até $600 \mathrm{~kg} \mathrm{ha}^{-1}$ de $\mathrm{K}_{2} \mathrm{O}$, mostrando uma relação adequada de 1,4 e 1,07 nas doses de 300 e $450 \mathrm{~kg} \mathrm{ha}^{-1}$, quando foram obtidas altas produções.

Quanto às relações $(\mathrm{Ca}+\mathrm{Mg}) / \mathrm{K}$ no solo, Mascarenhas et al. (1987) mostraram que as melhores relações estavam entre 23 e 31 para as altas produções de soja, quando o $\mathrm{K}, \mathrm{Ca}$, e $\mathrm{Mg}$ estão em concentrações adequadas. Na TABELA 2 observa-se que na dose de 300 $\mathrm{kg} \mathrm{ha}^{-1}$ de $\mathrm{K}_{2} \mathrm{O}$, as relações $(\mathrm{Ca}+\mathrm{Mg}) / \mathrm{K}$ no solo variaram de 21 (1992/93) a 25 (1993/94) e, na média dos dois anos, foi de 23 , coincidindo com as melhores produções obtidas na mesma dose na presença de calagem, segundo a análise conjunta dos três anos (TABELA 1). Como o valor médio para a relação $(\mathrm{Ca}+\mathrm{Mg}) / \mathrm{K}$ foi apenas 14 na dose de $450 \mathrm{~kg} \mathrm{ha}^{-1}$ de $\mathrm{K}_{2} \mathrm{O}$, deve ter ocorrido uma quantidade de potássio excedente no solo, mas que não afetou a produtividade de grãos, pelo fato do $\mathrm{K}, \mathrm{Ca}$ e $\mathrm{Mg}$ estarem em concentrações adequadas.

Entre os cultivares, o FT-2 respondeu com maior magnitude à adubação potássica, em forma de produção de grãos, sendo o efeito quadrático (TABELA 1). A máxima produtividade de $3048 \mathrm{~kg} \mathrm{ha}^{-1}$, em 1991/92, foi obtida com $599 \mathrm{~kg} \mathrm{ha}^{-1}$ de $\mathrm{K}_{2} \mathrm{O}$. Por outro lado, para o cultivar IAC17, a aplicação de doses de potássio mostrou efeito linear positivo, não sendo possível estimar o ponto de máxima eficiência técnica. No ano de 1992/93 (primeiro ano do efeito residual), houve resposta apenas para o cv. FT-2 e ela foi quadrática com a produção máxima de $2101 \mathrm{~kg} \mathrm{ha}^{-1}$ obtida com a aplicação de $349 \mathrm{~kg} \mathrm{ha}^{-1}$ de $\mathrm{K}_{2} \mathrm{O}$. O cultivar
TABELA 2 - Relação (Ca+Mg)/K no solo em função de doses de potássio em presença de todos níveis da calagem.

\begin{tabular}{cccc}
\hline $\mathrm{K}_{2} \mathrm{O}$ & $\begin{array}{c}1992 / 93 \\
(\mathrm{Ca}+\mathrm{Mg}) / \mathrm{K}\end{array}$ & $\begin{array}{c}1993 / 94 \\
(\mathrm{Ca}+\mathrm{Mg}) / \mathrm{K}\end{array}$ & Média \\
\hline$--\mathrm{kg} \mathrm{ha}^{-1}--$ & & & \\
0 & 105 & 244 & 175 \\
150 & 38 & 59 & 49 \\
300 & 21 & 25 & 23 \\
450 & 15 & 13 & 14 \\
600 & 12 & 8 & 10 \\
\hline
\end{tabular}

IAC-17 nesse ano não respondeu significativamente à adubação potássica. No terceiro ano (1993/94), correspondente ao segundo ano do efeito residual, os efeitos foram quadráticos. A produção do cv. FT-2 foi sempre superior ao cv. IAC-17. As maiores produtividades de grãos, de 2165 e $1825 \mathrm{~kg} \mathrm{ha}^{-1}$, seriam alcançadas com a aplicação de 473 e $404 \mathrm{~kg} \mathrm{ha}^{-1}$ de $\mathrm{K}_{2} \mathrm{O}$, respectivamente.

Na média dos três anos, a produção máxima de grãos, para o cv. IAC-17, foi de $1786 \mathrm{~kg} \mathrm{ha}^{-1} \mathrm{com}$ a aplicação de $370 \mathrm{~kg} \mathrm{ha}^{-1}$ de $\mathrm{K}_{2} \mathrm{O}$ (TABELA 1). Para o cv. FT-2, foram necessários $450 \mathrm{~kg} \mathrm{ha}^{-1}$ de $\mathrm{K}_{2} \mathrm{O}$ para uma produção de $2384 \mathrm{~kg} \mathrm{ha}^{-1}$. Para a dose ideal de potássio do cv. IAC-17 (370 kg ha-1 de $\mathrm{K}_{2} \mathrm{O}$ ) o cv. FT-2 produziu $2354 \mathrm{~kg} \mathrm{ha}^{-1}$, mostrando a sua eficiência na utilização do fertilizante e confirmando os resultados de Fernandes et al. (1993), que mostraram que o cultivar IAC-17 é eficiente em explorar o potássio disponível do solo enquanto, o FT2 é mais eficiente em utilizar o potássio aplicado.

\section{AGRADECIMENTOS}

A Ocimara A. Alves e Paulo E. Magalhães (pessoal de apoio) na execução do experimento no campo.

\section{REFERÊNCIAS BIBLIOGRÁFICAS}

ALVARENGA, M.I.N.; LOPES. A.S. Influência da adubação potássica no equilíbrio de cátions em latossolo roxo Distrófico, fase cerrado. Pesquisa Agropecuária Brasileira, v.23, p.347355, 1988.

BATAGLIA, O.C.; FURLANI, A.M.C.; TEIXEIRA J.P.F.; GALLO, J.R. Métodos de análise química de plantas. Campinas: IAC, 1983. 48p. (Boletim Técnico, 78).

BORKERT, C.M.; FARIAS, J.R.B.; SPOLADORI, C.L. Resposta da soja à adubação e disponibilidade de potássio em Latossolo Roxo distrófico. Pesquisa Agropecuária Brasileira, v.32, p.1235-1249, 1997.

FEHR, W.R.; CAVINESS, C.E.; BURMOOD, D.T.; PENNINGTON, J.S. Stage of development descriptions for soybeans, Glycine $\max ($ L.) Merrill. Crop Science, v.11, p.929-931, 1971.

FERNANDES, D.M.; ROSSETTO, C.A.V.; ISHIMURA, I; ROSOLEM, C.A. Nutrição da soja e formas de potássio no solo em função de cultivares e adubação potássica. Revista Brasileira de Ciência do Solo, v.17, p.405-410, 1993.

GARGANTINI, H.; COELHO, F.A.S.; SOARES, F.V.E. Levantamento de fertilidade dos solos do Estado de São Paulo. Campinas: IAC, 1970. 32p. 
GALLO, P.B.; MASCARENHAS, H.A.A.; QUAGGIO, J.A.; BATAGLIA, O.C. Resposta diferencial das culturas de soja e sorgo à calagem. Revista Brasileira de Ciência do Solo, v.10, p.253-258, 1986.

ITO, M.F; TANAKA, M.A.S.; MASCARENHAS, H.A.A.; TANAKA, R.T.; DUDIENAS, C.; GALLO, P.B. Efeito residual da calagem e de adubação potássica sobre a queima foliar (Cercospora kikuchii) da soja. Summa Phytopathologica, v.19, p.21-22, 1993.

ITO, M.F.; MASCARENHAS, H.A.A.; TANAKA, M.A.S.; DUDIENAS, C.; TANAKA, R.T.; GALLO, P.B.; MIRANDA, M.A.C. Efeito residual da adubação potássica e da calagem sobre a incidência de Phomopsis spp. em sementes de soja. Fitopatologia Brasileira, v.19, p.44-49, 1994.

JAWORSKI, C.A.; BARBER, S.A. Soil properties as related to potassium uptake by alfafa. Soil Science, v.86, p.37-41, 1957.

MASCARENHAS, H.A.A.; DEMATTÊ, G.D.; MIYASAKA, S.; IGUE, T. Estudos preliminares sobre a adubação econômica da soja (Glycine max L.) na região da Alta Mogiana em Latossolo roxo e Latossolo vermelho amarelo fase arenosa. Campinas: Instituto Agronômico, 1971. 7p. (Projeto BNDE/ANDA/CIA, 3).

MASCARENHAS, H.A.A.; GALLO, J.R.; RAIJ, B.; IGUE, T. \& BATAGLIA, O.C. Efeito da calagem nas características químicas do solo e na nutrição da soja em latossolo roxo distrófico de cerrado. Bragantia, v.35, p.273-278, 1976.

MASCARENHAS, H.A.A.; VALADARES, J.M.A.; ROTTA, C.L.; BULISANI, E.A. Adubação potássica na produção de soja, nos teores de potássio em Latossolo Roxo Distrófico de cerrado. Bragantia, v.40, p.125-134, 1981.

MASCARENHAS, H.A.A.; MIRANDA, M.A.C.; LELIS, L.G.L.; BULISANI, E.A.; BRAGA, N.R.; PEREIRA, J.C.V.N.A. Haste verde e retenção foliar em soja causada por deficiência de potássio. Campinas: IAC, 1987. 15p. (Boletim Técnico, 119).

MASCARENHAS, H.A.A.; PATRICIO, F.R.A.; TANAKA, M.A.S.; TANAKA, R.T.; PIANOSKI, J. Ocorrência de fungos em sementes de soja produzidas sob calagem e adubação potássica residuais. Scientia Agricola, v.52, p.426-430, 1995.
NELSON, W.L.; BARBER, S.A. Nutrient deficiencies in legumes for grain and forage. In: SPRAGUE, H.B. (Ed.) Hunger signs in crops. New York: D. McKay, 1964. p143-180.

RAIJ, B. van; MASCARENHAS, H.A.A. Calibração de potássio e fósforo em solos para soja. In: CONGRESSO BRASILEIRO DE CIÊNCIA DO SOLO, 15., Campinas, 1976. Anais. Campinas: Sociedade Brasileira de Ciência do Solo, 1976. p.309-315

RAIJ, B. van; QUAGGIO, J.A. Métodos de análise de solo para fins de fertilidade. Campinas: Instituto Agronômico, 1983. 31p. (Boletim Técnico, 81).

RITCHEY, K.D.; CERKAUSKAS, R.F.; SILVA, J.U.; VILELA, L. Residual effects of potassium and magnesium on soybean yield and on disease incidence in a cerrado dark red latosol. Pesquisa Agropecuária Brasileira, v.22, p.825-832, 1987.

ROSOLEM, C.A.; NAKAGAWA, J.; MACHADO, J.R. Adubação potássica da soja em Latossolo Vermelho Escuro fase arenosa. Pesquisa Agropecuária Brasileira, v.19, p.1319-1326, 1984.

ROSOLEM, C.A.; MACHADO, J.R.; MAIA, L.C.; NAKAGAWA, J. Respostas de soja ao magnésio do solo. Revista Brasileira de Ciência do Solo, v.16, p.47-54, 1992.

SCHERER, E.E. Resposta da soja à adubação potássica em Latossolo húmico distrófico num período de doze anos. Revista Brasileira de Ciência do Solo, v.22, p.49-55, 1998.

TANAKA, R.T.; MASCARENHAS, H.A.A. Soja, nutrição correção do solo e adubação. Campinas: Fundação Cargill, 1992. 60p. (Série Técnica, 7).

TANAKA, R.T.; MIRANDA, M.A.C.; MASCARENHAS, H.A.A.; CAMARGO, M.B. IAC aponta causas de prejuízos da safra 1992/93 da cultura da soja. O Agronômico, v.45, p.9-10, 1993.

USHERWOOD, N.R. Interação do potássio com outros íons. In: SIMPÓSIO SOBRE O POTÁSSIO NA AGRICULTURA BRASILEIRA, Londrina, 1982. Anais. Piracicaba: Instituto de Potassa e Fosfato, 1982. p.227-247.

YAMADA, T.; BORKERT, C.M. Nutrição e produtividade da soja. In: SIMPÓSIO SOBRE A CULTURA E PRODUTIVIDADE DA SOJA, Piracicaba, 1992. Anais. Piracicaba: USP, ESALQ; FEALQ, 1992. p.180-212.

Recebido em 23.06.99 